\title{
THE EFFECT OF MEASUREMENT GEOMETRY FOR ON-LINE TEXTURE ANALYZING SYSTEMS
}

\author{
P. BLANDFORD, and J.A. SZPUNAR \\ Department of Metallurgical Engineering, McGill University, \\ 3450 University St., Montreal, Quebec, H3A 2A7, Canada.
}

A laboratory prototype of a system for the measurement of texture online in the manufacturing process of steel or aluminum is being built at McGill University. In order to test the effect that the different measurement geometry, i.e an angular dispersive reflection or an energy dispersive transmission geometry, has on the calculation of the coefficients of the orientation distribution function (ODF), a number of simulated texture measurements were done for both geometries. The effect of the measured texture on the prediction of magnetic torque was also studied.

In general, there is good agreement between the textures calculated by simulating the two measurement geometries and the original texture used to recalculate the pole figures from which the simulation data was extracted. The results also showed that, for the slightly inhomogeneous steel, the bulk magnetic torque predicted from the texture measurements simulating the energy dispersive, transmission geometry was only slightly better than that predicted from the angular dispersive, reflection geometry. In the case of the inhomogeneous steel, the prediction from the transmission geometry was significantly better than that of the reflection geometry. It has been shown that measurement geometry for on-line texture analyzing systems has a significant impact on the accuracy of the texture information obtained and the prediction of certain property anisotropies, especially for those sheet materials exhibiting significant through-thickness inhomogeneity.

\section{INTRODUCTION}

The need for higher rolled sheet quality leads to a demand for greater knowledge of how processing affects sheet properties and qualities, for which sheet texture is an important parameter. Destructive techniques for obtaining such information are costly, both in terms of time and money. The use of on-line, non-destructive techniques for obtaining information of the sheet texture is quite rare ${ }^{1,2}$, and the texture information obtained is restricted to the intensities of selected diffraction maxima. While this is suitable for certain applications (quality control, etc), in the general case, 
more detailed information about the texture is warranted. It is the aim of the design group at McGill University to develop such quantitative, on-line texture analyzing systems.

Work on this system has been described previously $3,4,5,6$ and to date a laboratory prototype has been built. Recently, we have been carrying out various texture measurement simulations designed to increase our understanding as to how the on-line measurement geometry affects our ability to accurately calculate the coefficients of the ODF and to predict the anisotropies of certain important physical and material properties.

\section{DESCRIPTION OF TEXTURE MEASUREMENT SIMULATIONS}

Physically, the on-line texture measurement system consists of an Xray tube and generator and an INEL CPSD $120^{\circ}$ position sensitive detector hung over the sheet from a frame, and/or two germanium detectors located under the sheet surface 5,6 . The tube and detectors are arranged with respect to each other such that the position sensitive detector records up to 14 diffraction maxima in a reflection geometry while the energy dispersive detectors record 11 maxima in a transmission geometry. By keeping the position of all detectors fixed with respect to the incident $X$-ray beam, the intensities along certain lines in the relevant pole figures can be measured by rotating the equipment by 180 degrees about the sheet normal.

In the simulation of the texture measurements, the pole figure coordinates of the diffracted planes relevant for steel were calculated for both geometries using a molybdenum x-ray source powered by a $60 \mathrm{kV}$ generator. The intensity at each of these points was obtained by recalculating the relevant pole figures using up to $1=22$ coefficients of the ODF $\left(\mathrm{C}_{1}{ }^{\mu v}\right)$ taken from previous analysis made by measuring three incomplete pole figures in the traditional way.

The reduced pole figure data sets were then used as inputs to a computer program 4 which uses a least squares fit of calculating the coefficients of the ODF from sparse pole figure data. Simulations of both measurements geometries were carried out on two different ferrosilicon steels, one exhibiting marked through-the-sheet-thickness inhomogeneity of texture and one which did not. The inhomogeneity of these steels have been studied previously7,8 allowing pole figures for each of the two steels to be calculated using coefficients, $\mathrm{C}_{1}{ }^{\mu \mathrm{v}}$, from the surface texture measurements for the reflection geometry simulation, and through-thickness-averaged coefficients of the texture for the transmission geometry simulations.

\section{RESULTS AND DISCUSSION}

Tables 1 and 2, respectively present a comparison between the coefficients of the ODF resulting from the reflection and transmission geometry simulations to the ODF coefficients used to calculate the pole figure data used in the simulations, for the Steel A (slightly inhomogeneous) and Steel B (inhomogeneous). 
The results for the Steel $A$, with the notable exception of the $C_{6}{ }^{14}$ coefficients for the reflection simulation and the $\mathrm{C}_{6}{ }^{12}$ coefficient for the transmission measurement simulation show that the least squares fit gives coefficients which reasonably describe the original texture. When comparing the coefficients calculated from the reflection geometry simulation to those from the transmission geometry simulation, the significant differences found seem to indicate that the texture in this steel is quite inhomogeneous, contrary to what is known (as shown by a comparison of the original coefficients of the surface texture to those of the throughthickness averaged coefficients).

The coefficients presented in Table 2 for the inhomogeneous Steel B show a much better agreement with those of the original. In this case, the simulations have accurately obtained the ODF coefficients, and thus preserved the information of the inhomogeneity of texture observed in this steel. Comparing the coefficients of the ODF is not the only, or most useful, method whereby one can assess the effect that measurement geometry has on the texture measurements.

Another method, and one particularly relevant to on-line texture analyzing systems, is to use the calculated coefficients to predict the anisotropy of a physical property and then to compare the quality of the predictions with those from the original $\mathrm{C}_{1}{ }^{\mu v}$. Using a well established5,9,10 correlation between the texture and the anisotropy of magnetocrystalline energy ${ }^{11}$, the magnetic torque can be calculated and decomposed into its Fourier components $\sin 2 \beta, \sin 4 \beta$ and $\sin 6 \beta$ (where $\beta$ is the angle from the sheet rolling direction). The Fourier coefficients $\mathbf{a}_{2}, \mathbf{a}_{4}$, and $\mathbf{a}_{6}$ were calculated using the coefficients of the ODF presented in Tables 1 and 2. Figures 1 and 2, respectively, present the Fourier coefficients of the magnetic torque predicted for Steel A and Steel B.

Figure 1a shows that the Fourier coefficients calculated from the original texture coefficients and those from the simulations are within error

Table 1: Comparison of ODF coefficients calculated in the various simulations for Steel $A$.

\begin{tabular}{|c|c|c|c|c|}
\hline \multicolumn{5}{|c|}{ various simulations for Steel A. } \\
\hline $\mathrm{C}_{1}{ }^{\mu v}$ & $\begin{array}{c}\text { Surface } \\
1_{\max }=22\end{array}$ & $\begin{array}{c}\text { Sim. } \\
\text { Reflect. }\end{array}$ & $\begin{array}{c}\text { Average } \\
\mathrm{l}_{\max }=22\end{array}$ & $\begin{array}{c}\text { Sim. } \\
\text { Trans. }\end{array}$ \\
\hline $\mathrm{C}_{4}{ }^{11}$ & 0.75 & 1.06 & 0.41 & 0.51 \\
\hline $\mathrm{C}_{4}{ }^{12}$ & -1.34 & -1.37 & -0.81 & -0.47 \\
\hline $\mathrm{C}_{4}{ }^{13}$ & -1.39 & -1.12 & -1.54 & -1.38 \\
\hline $\mathrm{C}_{6}{ }^{11}$ & 2.82 & 3.28 & 2.94 & 2.71 \\
\hline $\mathrm{C}_{6}{ }^{12}$ & -3.46 & -3.80 & -3.15 & -2.23 \\
\hline $\mathrm{C}_{6}{ }^{13}$ & 1.49 & 1.31 & 1.11 & 0.56 \\
\hline $\mathrm{C}_{6}{ }^{14}$ & -0.38 & -1.35 & -0.19 & 0.10 \\
\hline
\end{tabular}


$( \pm 15 \%)$ of each other. The difference in the $a_{2}$ and $a_{4}$ Fourier coefficients calculated from the reflection and transmission simulations is significant and indicates the extent of the inhomogeneity observed in Steel A. The variation shown in the $a_{6}$ coefficient is even greater, but is to be expected because its magnitude depends only on the $\mathrm{C}_{6}{ }^{14}$ ODF coefficients and can fluctuate significantly.

The results presented in Figure $1 \mathrm{~b}$ show that the significant inhomogeneity of Steel B exhibits itself by a marked difference between the Fourier coefficients calculated from the reflection geometry simulations and those calculated from the transmission geometry simulations. The magnitude of the sin $2 \beta$ component, $a_{2}$, calculated for the reflection geometry is $a_{2}=(-1.7 \pm 15 \%) \times 10^{4} \mathrm{ergs} / \mathrm{cm}^{3}$, while that for the transmission geometry simulation is $a_{2}=(-2.4 \pm 15 \%) \times 10^{4} \mathrm{ergs} / \mathrm{cm}^{3}$; the magnitude of the $\sin 4 \beta$ component is $a_{4}=(-1.3 \pm 15 \%) \times 10^{4} \mathrm{ergs} / \mathrm{cm}^{3}$ and $a_{4}=(-2.4 \pm 15 \%) \times 10^{4}$ ergs $/ \mathrm{cm}^{3}$, respectively, for the reflection and transmission geometry simulations. The good agreement between the original and simulated ODF coefficients shown in Table 2 is similarly shown by the quality of agreement between the corresponding Fourier coefficients.

The magnetic torque of the two steels used in this paper has been measured previously, and the effect that the observed textural inhomogeneity has on its correlation with the texture has been studied7,8. The method of analysis used was based on a comparison of the Fourier coefficients calculated from the experimental and predicted torque curves. The fifth bar in the bar graphs presented in Figures $1 \mathrm{a}$ and $1 \mathrm{~b}$, respectively, represents the magnitude of the appropriate Fourier coefficients of the experimentally measured torque curves for Steel A and Steel B. The results of the previous study has shown that of the $a_{2}, a_{4}$ and $a_{6}$ Fourier coefficients, only the $a_{4}$ coefficient (determining the magnitude of the $\sin 4 \beta$ components of the experimental torque), is free from various experimental errors.

Table 2: Comparison of ODF coefficients calculated in the various simulations for Steel B.

\begin{tabular}{|c|c|c|c|c|}
\hline $\mathrm{C}_{1}{ }^{\mu v}$ & $\begin{array}{c}\text { Surface } \\
\mathrm{l}_{\max }=22\end{array}$ & $\begin{array}{c}\text { Sim. } \\
\text { Reflect. }\end{array}$ & $\begin{array}{c}\text { Average } \\
\mathrm{l}_{\max }=22\end{array}$ & $\begin{array}{c}\text { Sim. } \\
\text { Trans. }\end{array}$ \\
\hline $\mathrm{C}_{4}{ }^{11}$ & -0.11 & -0.12 & -0.35 & -0.29 \\
\hline $\mathrm{C}_{4}{ }^{12}$ & -1.44 & -1.43 & -2.05 & -2.10 \\
\hline $\mathrm{C}_{4}{ }^{13}$ & 0.34 & 0.40 & 0.79 & 0.76 \\
\hline $\mathrm{C}_{6}{ }^{11}$ & 1.90 & 2.10 & 1.06 & 0.97 \\
\hline $\mathrm{C}_{6}{ }^{12}$ & -1.95 & -1.95 & -1.27 & -1.43 \\
\hline $\mathrm{C}_{6}{ }^{13}$ & 1.06 & 0.85 & 0.98 & 0.92 \\
\hline $\mathrm{C}_{6}{ }^{14}$ & 0.53 & 0.31 & 0.77 & 0.69 \\
\hline
\end{tabular}




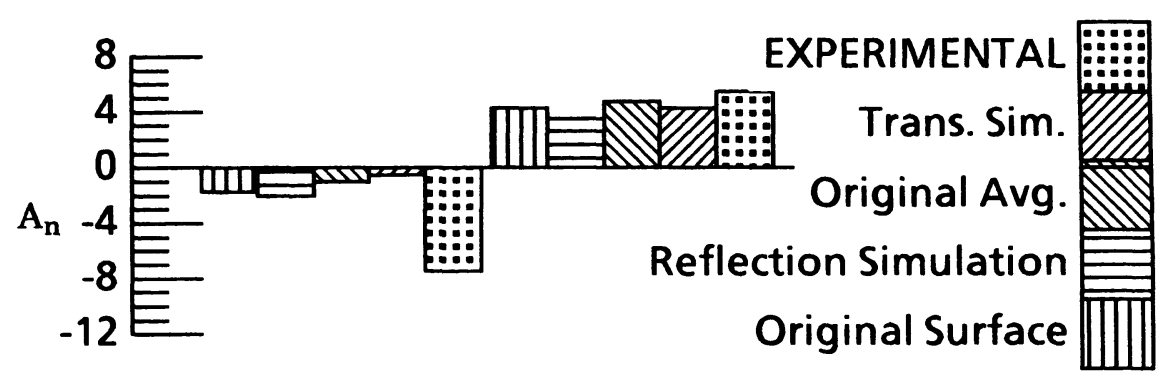

a)

A2

A4

\section{FOURIER COEFFICIENT}

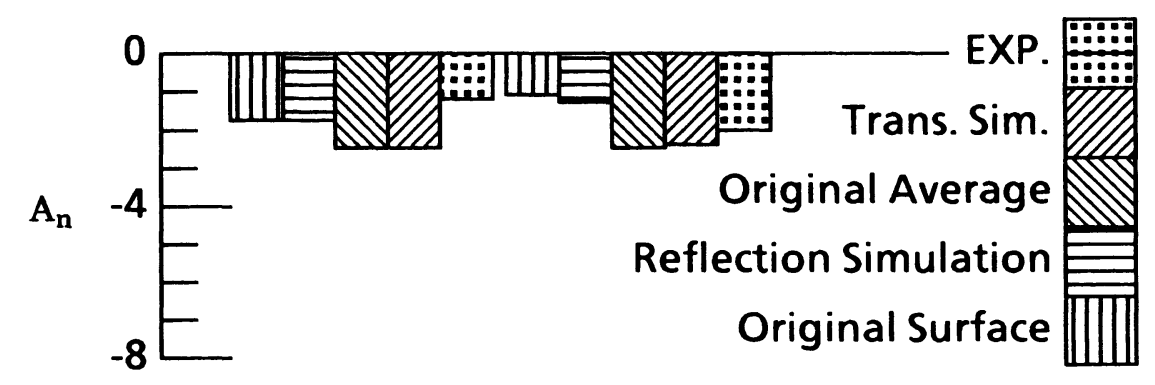

b)

A2

A4

Figure 1: Fourier coefficients, $A_{n}$, of magnetic torque $\left(/ 10^{4} \mathrm{ergs} / \mathrm{cm}^{3}\right)$ for a) Steel A, and b) Steel B.

A comparison of the $a_{4}$ coefficients for the Steel A (Figure 1a) shows that generally, the coefficient of the experimental torque curve is somewhat higher than those resulting from either of the two simulations; however, the coefficients resulting from the transmission geometry simulation, i.e the coefficient representing the torque predicted from the average or bulk texture, is closer to the experimental result than the coefficient resulting from the reflection geometry simulation.

A similar comparison of the magnitudes of the $\sin 4 \beta$ components of Steel B (Figure 1b) shows that only the Fourier coefficient resulting from the averaged, transmission geometry simulation agrees within error with the experimental result, and clearly indicates, for this case, the ability of an online system using this geometry to predict the bulk magnetic torque; the $a_{4}$ coefficient from the reflection geometry is much smaller.

\section{CONCLUSION}

This work reports some of the results we have obtained while developing on-line texture measurement and analysis systems. In this paper, the effect of measurement geometry on the accurate measurement of texture and the prediction of material anisotropy were studied through simulating two different (one reflection, one transmission) on-line texture 
analyzing systems. The simulations were carried out on two steels exhibiting different amounts of textural through-thickness inhomogeneity, and the impact of the measurement geometry was studied by: 1) comparing calculated coefficients of the ODF, 2) comparing the Fourier coefficients of the magnetic torque predicted using a correlation derived by Bunge ${ }^{11}$, and 3) by comparing the Fourier coefficient resulting from the predictions to those from experimentally measured torque curves.

In general, the agreement between the ODF coefficients calculated from the simulations with the ODF coefficients used to recalculate the pole figure data, are good, although certain coefficients show marked differences. In the case of the slightly inhomogeneous steel (Steel A), the inhomogeneity was difficult to see by comparing the ODF coefficients. However, the inhomogeneity became more apparent when comparing the Fourier coefficients of the magnetic torque predicted from the ODF coefficients. This is due to the fact that the predictions of the Fourier coefficients generally involves a number of ODF coefficients and therefore are not as susceptible to large errors in specific ODF coefficients. The comparison of the predicted Fourier coefficients showed that the impact of the measurement geometry on the analysis is most strongly felt in the steel exhibiting significant inhomogeneity.

AKNOWLEDGMENTS: The authors would like to thank the National Science and Engineering Research Council of Canada and the Ministry of Education of Quebec (FCAR) for the funding of this work. P. Blandford would like also to thank Stelco Inc. of Canada for his scholarship.

\section{REFERENCES}

1 H.J. Kopineck, Experimental Techniques of Texture Analysis, Ed. by H.J. Bunge, DGM Informationsgesellschaft Verlag (1986).

2 C.O. Ruud, J.C. Conway, K. Kozaczek and C-J. Yu, 16th Annual NSF Grantees Conf., Arizona State U., Tempe, Jan 8-12 (1990).

3 J.A. Szpunar, Textures of Crystalline Solids, 4, 171(1981).

4 J.A. Szpunar and D.C. Hinz, J. of Mat. Sci., 25, 2846 (1990).

5 P. Blandford and J.A. Szpunar, Proc. of the World Materials Congress, Chicago (1988).

6 P. Blandford and J.A. Szpunar, Proc. of QNDE Techniques, Brunswick, Maine (1989).

8 P.Blandford, M. Eng Thesis, McGill University (1989).

9 P.Blandford and J.A. Szpunar, IEEE Trans. on Magnetics, to be published (1990).

10 J.A. Szpunar and M. Ojanen, Met. Trans. AIME, 6A, 561 (1975).

11 W.B. Hutchinson and J.G. Swift, Texture, 1, 117 (1973).

12 H.J. Bunge, Texture Analysis in Materials Science, Butterworths, London (1982). 